\title{
Le discours des commentaires sur le football des internautes lecteurs de www.lequipe.fr
}

\author{
Discursive features of web-users commentaries \\ at the football newsgroup of the www.lequipe.fr
}

\author{
Andrzej Napieralski \\ Université de Łódź \\ napieralski.a@op.pl
}

\begin{abstract}
Internet visitors, having found this instrument of global communication, have discovered a new field of verbal battles where they can release their emotions, expressing completely anonymously views. The Internet has changed the image of debate, and of polemic discussion, which earlier was an exchange of replies, and now thanks to forums and "reactions", a "bath of opinions" has manifest, where everyone can add his two cents. The commentary we would like to present in this work are the reactions of the readers of the website www.lequipe.fr who after each article had the opportunity to express their arguments and their reactions to the article they had read.
\end{abstract}

Keywords: sociolinguistics, lexicology, youth language, linguistics, football, discourse analysis

«La fonction essentielle de cet instrument qu'est une langue est celle de communication : le français par exemple, est avant tout l'outil qui permet aux gens "de langue française" d'entrer en rapport les uns avec les autres" (Martinet, 1996 : 9). Il faut signaler que depuis l'an 2000, la communication entre «les uns avec les autres » se fait souvent via Internet. Cet outil de communication globale existant depuis 1990 facilite l'extraversion et en conséquence, l'échange d'opinions entre les gens qui souhaitent s'exprimer sur n'importe quel sujet. La structure sociale a changé et elle repose aujourd'hui sur le réseau global qui répond aux besoins de liberté des individus et de l'expression. On souhaiterait que cette liberté soit utilisée pour une bonne cause, d'une manière démocratique et sans répercutions, cependant elle rappelle de plus en plus ce droit naturel décrit par Thomas Hobbes dans le Léviathan où la règle 
bellum omnium contra omnes ${ }^{1}$ semble prendre le dessus. L'utilisation du mot "liberté" dans le contexte des échanges verbaux sur le web est très souvent dépravée, ça devient plutôt le droit à la polémique virulente synonyme de guerre à arguments où tous les coups sont permis. Les internautes s'escriment de la liberté comme d'un droit héréditaire à la manière du premier amendement de la Constitution des Etats-Unis qui assure la liberté de la parole et de la presse. La "guerre" des internautes a pour enjeu d'influencer les autres, d'attirer l'attention, d'inspirer et surtout de forcer à réagir en soutenant ou contestant les propos proférés. Les terrains des échanges sont divers, cela peuvent être les ( $t$ )chats, les forums, les blogs, les réseaux sociaux, mais aussi les endroits prévus pour, qui se situent sous les articles et qu'on appelle les commentaires. La possibilité qui est donnée à chacun pour ajouter son grain de sel à une discussion peut être comparée, toutes proportions gardées, à l'agora du polis d'Aristote qui était selon celui-ci un élément essentiel d'un monde civilisé. Internet a bouleversé le système de valeurs qui s'établissait depuis des siècles, le respect pour l'éducation, la culture et les traditions a disparu au moment où a apparu la possibilité pour Monsieur tout le monde de devenir expert dans chaque matière. Les autorités, ce ne sont plus les universitaires, les gens diplômés, cultivés, chevronnés, aujourd'hui on écoute ceux qui savent flatter nos goûts et nos convictions afin d'obtenir le plus grand nombre de "like" sur Facebook. Dans le cadre de ce travail, ce sont les commentaires situés sous les articles de la presse sportive et plus particulièrement, ceux traitant du football qui se trouvent sur le site Internet du magazine sportif L'Equipe (www.lequipe.fr). La méthodologie appliquée à ce travail s'inspire de la rhétorique aristotélicienne tripartie (qui est divisée en ethos, logos, pathos). Tout d'abord, nous prendrons en considération la façon dont se construit l'ethos des internautes postant leurs commentaires. Ensuite, nous nous focaliserons sur le problème des effets pathémiques du discours des internautes qui par leurs productions tendent à influencer les lecteurs de leurs posts ${ }^{2}$; dans ce but ils utilisent différents instruments langagiers qui seront analysés à la suite de cet article.

Ce qui est caractéristique pour ces commentaires, c'est la façon dont sont exprimés (adaptés au lieu de l'échange) différents types d'opinions et surtout les émotions qu'elles véhiculent. Comme l'a bien remarqué Chaïm Perelman : «Un argument, s'il n'est pas adapté à l'auditoire, peut susciter une réaction négative. S'il suscite des objections qui l'emportent dans l'esprit des auditeurs, l'argument paraitra faible, et cette faiblesse peut rejaillir sur l'ensemble du discours, car l'image de l'orateur, ce qu'Aristote a qualifié d'ethos oratoire, en sera altérée...

\footnotetext{
${ }^{1}$ fr. la guerre de tous contre tous.

${ }^{2}$ Il s'agit de l'emprunt à l'anglais, substantif dérivé du verbe to post 'publier', néologisme répandu grâce au réseau social Facebook qui désigne entre autres un commentaire sur Internet.
} 
[...]» (Perelman, 2009: 174). Les arguments construisent et enchaînent les polémiques autour des sujets présents sur le site www.lequipe.fr, les internautes ne manquent pas à exprimer leurs réactions négatives. Selon l'approche de Dominique Maingueneau «L'ethos [du locuteur] est [...] attaché à l'exercice de la parole, au rôle qui correspond à son discours, et non à l'individu 'réel' [...] : c'est donc le sujet d'énonciation en tant qu'il est en train d'énoncer qui est ici en jeu » (Maingueneau, 1993 : 138). Ainsi, l'individu qui émet son opinion est perçu uniquement selon les propos qu'il profère et non pas par sa prestation oratoire. L'ethos oratoire de chaque participant se forme donc au fil de la discussion, quoiqu'il soit vrai en même temps que la qualité des énonciations varie en fonction de la position sociale du locuteur et de l'éducation qu'il a poursuivie.

Il faut souligner que tous les articles ne suscitent pas autant d'intérêt de la part des internautes, le sujet de l'article et le protagoniste mentionné étant les facteurs décisifs pour attirer l'attention des gens qui commentent.

Les commentaires que nous voudrions présenter et analyser dans cet article sont les réactions des lecteurs du site Internet www.lequipe.fr, qui après chaque article ont la possibilité d'exprimer leurs arguments et leurs réactions. Pour les besoins de notre analyse, nous avons retenu les textes suivants :

1) Anelka, deux buts, une quenelle (28 décembre 2013)

2) Dieudonné interdit sur le territoire (3 février 2014)

3) Anelka quitte West Brom! (14 mars 2014)

4) ,Quenelle”, Nasri s'explique à son tour (30 décembre 2013)

5) Blanc est intransigeant ( 7 mars 2014)

6) PSG-OM : grand classique à l'audimat (3 mars 2014)

7) Matuidi : «Un gros coup» (3 mars 2014)

8) Gignac a été cambriolé (3 mars 2014)

9) Ribéry et Benzema sauront à 13 h30 (30 janvier 2014)

10) Zahia: Benzema et Ribéry absents (20 janvier 2014)

11) Ribéry et Benzema comparaitront (14 août 2013)

12) Ribéry demande le huis clos (16 juin 2013)

Un sujet strictement sportif n'incite pas les lecteurs à la polémique autant qu'un sujet contestable ou à scandale. L'idée principale de commenter un article sportif est avant tout de donner des précisions sur le contenu, de corriger un lapsus ou une information fautive. S'il est vrai que cela se rapporte aux articles à teneur neutre, il n'est pas moins vrai que les articles qui dérivent du contenu essentiellement sportif encouragent les lecteurs à la discussion, voir parfois même à la querelle. Ce qui est le plus discutable, ce sont les goûts individuels comme : le choix d'un club, la sympathie envers un personnage, l'indignation qui naît à l'encontre d'un sujet. Un commentaire qui comporte en soi une dose trop importante de "vérité » subjective donne naissance à un débat qui se 
transforme souvent en échange de joutes oratoires. Les footballeurs de l'équipe de France (Nicolas Anelka, Samir Nasri, Frank Ribéry et Karim Benzema), mis à part leurs performances sportives, participent souvent à des situations honteuses qui font que les gens ne les jugent plus grâce à leurs attributs sportifs, mais en fonction de la «gaffe » qu'ils ont commise. Les affaires dans le football ne sont pas rares et elles constituent le plus fréquent semeur de zizanie parmi les supporters et les fans de sport.

L'affaire Zahia ${ }^{3}$ est un scandale qui a impliqué deux joueurs de l'équipe de France de football (à savoir Franck Ribéry et Karim Benzema) accusés d'avoir eu recours aux services d'une jeune prostituée mineure Zahia Dehar. Ils ont finalement été relaxés, mais le procès a duré 4 ans et a suscité de nombreux débats et commentaires. L'affaire de la quenelle ${ }^{4}$ est un scandale avec l'humoriste Dieudonné M'bala M'bala dans le rôle principal. Cet artiste a propagé un geste (appelé par la suite la quenelle) qui consiste à tendre un bras vers le bas tout en posant la main de l'autre bras sur l'épaule. Ce geste est devenu très controversé du fait de son prétendu caractère antisémite. Certains joueurs pour montrer «leur solidarité avec l'humoriste» ont effectué une quenelle en célébrant un but. Aussi bien les acteurs de « l'affaire Zahia » que les « solidaires à Dieudonné » sont devenus la cible de critiques des médias de masse ainsi que des commentaires des internautes.

La pomme de discorde entre les gens qui postent sur le site lequipe.fr, ce sont aussi les préférences pour différents clubs de foot, en particulier pour le Paris Saint-Germain et l'Olympique de Marseille ${ }^{5}$. L'animosité entre ces supporters ne date pas d'hier et elle contribue fortement à l'élargissement du corpus de commentaires sur la toile.

Parmi les nombreux énoncés laissés par les adeptes du football sous les articles d'Internet, il a été recueilli un certain nombre de figures du discours que nous traiterons en citant Ruth Amossy: «dans la perspective qui nous intéresse... », du fait que « le catalogage n'a qu'une importance secondaire. Les figures sont des formes verbales dont il s'agit d'étudier la valeur argumentative en contexte » (Amossy, $2006: 202$ ). Ainsi parmi les post ${ }^{6}$ qui ont été repérés sur le $W e b$ nous trouvons des figures de pensée telle l'ironie.

13) pelĩ $\odot 14$ mars à $18: 50^{7}$

Anelka the king. T'es un homme reste digne. Tes ancêtres peuvent être fière de toi

\footnotetext{
${ }^{3}$ Les articles 9, 10, 11 et 12 traitent de ce sujet.

${ }^{4}$ Les articles 1, 2, 3, 4 sont liés à cette affaire.

${ }^{5}$ Les articles 5, 6, 7, 8 parlent des membres des clubs en question (entraîneurs ou joueurs).

${ }^{6} \mathrm{Il}$ s'agit de l'emprunt à l'anglais, substantif dérivé du verbe to post 'publier', néologisme répandu grâce au réseau social Facebook qui désigne entre autres un commentaire sur Internet.

${ }^{7}$ Les commentaires sont construits de sorte que nous retrouvons d'abord le nom (le blase, le pseudo) de la personne qui écrit : pelã $\odot$, ensuite il y a le jour et l'heure de la publication de ce
} 
14) Chabertttt 31 déc. à 12:48

Il a une faute sur le deuxième tweet, il a écrit: „mais je présente mes excuses a toutes les personnes que j'ai pu offenser”. La faute est sur le „a” c'est un „a” avec un accent donc un A comme ça , „à”.

paris c'est pourri 31 déc. à 13:50

Ah bin c'est cool sa heureusement que tu étais la parce que sinon je sais pas comment on aurai fait

15) damien1979 28 déc. à 15:07

Il aura décidément été au top de l'intelligence humaine durant toute sa carrière...

16) pihlness 28 déc. à 20:25

Tu m'as démasqué, désolé désolé désolé pour cette allusion inacceptable, je m'excuse, je m'agenouille, je rampe, je m'enfouis sous terre. Combien je dois?

17) Bouboune 3 mars à $8: 30$

Marseille, un club et une ville qui font rêver!

L'ironie qui est présente dans les commentaires est dirigée soit envers le protagoniste de l'article (exemples 13, 15 et 17) soit envers d'autres internautes (exemples 14, 16). Son utilisation se manifeste dans l'utilisation d'une antiphrase qui vise à ridiculiser quelqu'un (Anelka the king) ou quelque chose (Marseille, un club et une ville qui font rêver!). Il n'est pas rare de rencontrer une réplique ironique face à un commentaire qui était inutile ou inapproprié (le cas de la réplique de paris c'est pourri à Chabertttt dans l'exemple 14). L'ironie peut aussi intervenir dans la même phrase qu'une hyperbole (exemple 16) consistant à ajouter des propos exagérés d'une réalité. Dans l'exemple qui suit (exemple 18), il s'agit d'une énumération faite par les internautes de types de quenelles qui peuvent exister. La différenciation de types de ce geste est le résultat d'un jeu pervers consistant à se moquer de l'absurdité de la situation existante. L'hyperbole est parfois utilisée pour parler d'une personne, ainsi l'exemple 19 ridiculise le tennisman Rafael Nadal en se moquant de son comportement un peu trop turbulent sur le court.

18) mcoxy 28 déc. à 19:46

la tu es gourmand!!! une de 175 c'est sportif et c'est encore dans le cadre des conventions de Genève.

pelĩ̄ 28 déc. à 14:52

Il aurait pu faire une épaulée !!!

4.1.3.2 28 déc. à 15:38

C'est de l'artisanal celle là ! Lol

post : 14 mars à 18:50 et au-dessous, il y a le message (le contenu) transmis par l'auteur : Anelka the king. T'es un homme reste digne. Tes ancêtres peuvent être fière de toi. Il est important d'ajouter ici que les commentaires n'ont pas été modifiés et restent sous une forme qui ne correspond pas toujours aux normes du « français standard ». 
19) buratino 31 déc. à 12:15

Et Nadal, avec tous les gestes qu'il fait, il devrait être à Guantanamo

Le discours des commentaires des internautes repose souvent sur l'idée de l'échange verbal. Celui-ci est le plus souvent une réplique à un post antérieur qui enchaîne ensuite une discussion construite à base du modèle question > réponse ou attaque > réplique. Les échanges ont différents buts, ils peuvent être d'ordre informatif quand on tient à préciser une information relevant de l'histoire du football ou des règles de ce jeu; ou d'ordre polémique quand ils tiennent à mener une confrontation d'arguments.

20) XQuentinoX 28 déc. à 15:15

C'est pas du tout un geste antisémite! Faut arrêter de raconter n'importe quoi !

chingaos 28 déc. à 16:58

et la marmotte elle emballe ton bras avec le chocolat ?? comme vous êtes crédules...

AnTiX 28 déc. à 17:17

C'est toi qui est crédule à suivre l'avis des médias à ce sujet...

XTC 29 déc. à 21:16

C'est ça et la shohananas c'est un nouveau fruit? Quel hasard!

21) ioRek 7 mars à $15: 46$

Vous avez une put... d'équipe, avec des put... de joueurs, mais non il y en a ils ne peuvent pas s'empêcher de parler de l'OM. Il était temps que le Qatar fasse quelque chose de votre club... quel complexe vous aviez jusqu'en 2011 c'est dingue!!

elkikito turchinu 7 mars à 15:58

Un traître de quoi ??? Même si je déteste le PSG, quand on te propose une telle équipe faut être complètement débile, stupide, ignare ou simplement incompétent pour refuser !!!!! Y a qu un marseillais pour ouvrir sa bouche sans réfléchir !!!!!

L'exemple 20 est un échange de propos au sujet de la fameuse quenelle construit à la base d'un argument qui n'a pas plu à l'auditoire (C'est pas du tout un geste antisémite!) et qui a suscité une querelle au sujet de la signification de ce geste, qui est difficile à donner d'une façon objective. L'échange 21 est un exemple de querelle entre supporteurs des deux plus grands clubs adverses de la ligue 1. Ce «dialogue » repose sur l'histoire de l'interception du club de Paris par les Qatariens et le début des succès de ce club qui ne plaisent par forcément aux Marseillais.

22) doudou73800 14 août à 15:56

...pour une fois que les joueurs de l'EDF jouaient collectifs sur un si petit carr? de pelouse...

hugo101 14 août à $16: 14$

Bien d'accord pour une fois que ca fesait tourn?...

Vic Mc Kay 14 août à 16:29

ouais...ou ,jeu en triangle”. 
slyflow 14 août à 16:44

et il prenait du plaisir a jou? sur ce gazon

Passe-passe 14 août à 16:59

Et qu'ils en mettaient une au fond!!!

Un type d'échanges très intéressant qui a été remarqué dans les « réactions », c'est l'enchaînement des commentaires. Ce phénomène est un acte spontané qui consiste à réagir à l'énoncé précédent, ou au premier énoncé qui a inspiré cet échange. L'idée principale de cet enchaînement est librement liée avec le contenu de l'article, le but est de poursuivre la discussion en écrivant un post qui sera adapté à tout l'échange du point de vue de son message et de son caractère moqueur et persifleur. L'exemple 22 se reporte à 1 'affaire Zahia, les internautes écrivent des commentaires qui ont pour but de comparer l'aventure sexuelle des joueurs de l'équipe de France (EDF) au jeu de l'équipe. Les métaphores telles que "un petit carré de pelouse ", "ça faisait tourner", "jeu en triangle », «prendre du plaisir à jouer», «mettre une au fond» sont des locutions qui fonctionnent dans le jargon du football, mais elles peuvent se référer aussi à un rapport sexuel voire à une débauche collective. La fonction ludique joue un rôle important dans ce type d'énonciations, il ne s'agit plus dans ce cas là d'informer, mais de s'amuser.

23) JUIFARABE 3 févr. à 15:29

Quand on voit ce qu'on voit, on est bien content de penser ce qu'on pense.

kalotin 3 févr. à 15:42

Surtout quand on sait ce qu'on sait...

JUIFARABE 3 févr. à 16:48

...qu'on lit ce qu'on lit et qu'on entend ce qu'on entend. Enfin je me comprends.

yeuyeu 4 févr. à 15:24

la verite est ailleurs...

Le ludisme est présent dans certains commentaires, notamment dans ceux qui relèvent de l'absurde. Il arrive qu'un énoncé devienne la source d'inspiration pour d'autres internautes, notamment dans l'exemple 23, l'auteur du premier post a inspiré d'autres utilisateurs du site à se prononcer dans le même registre d'une constatation redondante où c'est la forme qui l'emporte sur le message. La dernière phrase de cet échange résume très bien le tout, la vérité est ailleurs, la fameuse devise de la série sur les phénomènes paranormaux X-files: Aux frontières du réel complète le ridicule qui émane de l'échange.

24) squadra azzura38 14 mars à 19:53

c pas parce que toi tu as du regarder la définition d'intégrité dans le dico que lui ne c'est pas ce que ça veut dire...

proximus2106 15 mars à 0:15

apprends déjà à écrire sans faute d'orthographe et après on pourra discuter... 
25) yannouch62 30 déc. à 19:18

Et si ses propres supporters faiser comme lui (être anti système) il ne gagnerais plus un rond!

DZ-Force 30 déc. à 19:39

Et si tu commençais par acheter un Bescherelle?

26) cheated 28 déc. à 19:20

Petit rappel: Messi et CR7 ont 32 Anelka dans chaque orteil et une vanne des inconnus ou de Coluche vaut plus que toute la carrière de dieudonné bref arrêtons de parler de gens médiocres qui n'en valent pas la peine. anelka et dieudonné en L2 avec sochaux et valenciennes et vite.

DJLLOSC 28 déc. à 19:33

Arrête un peu avec Sochaux et Valenciennes..Retourne à ta PS 3 !

hamozizeropocket 28 déc. à 23:42

Vu le nombre de pousse rouge, je pense que tu devrai aussi y aller en Ligue2

Les commentaires des internautes entraînent souvent des répliques inattendues qu'on pourrait qualifier d'《attaques ». La personne qui poste son message peut obtenir une réponse offensante résultant de la mauvaise volonté de celui qui réplique. L'exemple 24 illustre une situation dans laquelle un internaute se fait réprimander (apprends déjà à écrire sans faute d'orthographe) pour une remarque désobligeante envers un autre internaute (c pas parce que to $i$ tu as du regarder la définition d'intégrité dans le dico que lui ne c'est pas ce que ça veut dire). Dans ce cas nous avons affaire à une tentative de défense d'un opprimé par une attaque (proximus2106 attaque squadra azzura38 en défendant la personne visée par celui-ci). Cette condition ne doit pas toujours être remplie, il arrive souvent que l'attaque soit motivée par la simple aversion pour un post ou pour un internaute (exemple 25). Un commentaire dont les arguments sont manqués (le cas de 26) est le plus souvent confronté à de nombreuses répliques en forme d'attaque comme Retourne à ta PS $3^{8}$ qui implique que l'auteur du post est un enfant ou tu devrai aussi y aller en Ligue2 qui montre un désaccord total avec la constatation du prédécesseur.

27) oli54 16 juin à 10:26

trop envi que sa requête soit refusé, que l'on rigole un peu

ryu69100 16 juin à 10:57

Ha bon et pourquoi?

GregM34 16 juin à 11:21

ha bon et pourquoi pas?

28) Rojox 28 déc. à 14:50

Faut même pas chercher à polémiquer,c'est un gars qui fait plus rien et qui a besoin de provoquer pour qu'on parle de lui, il attend que ça

\footnotetext{
${ }^{8}$ La console de jeu de Sony.
} 
29) SpeedTrolling 3 mars à 10:05

Il y avait une équipe en face de Paris hier soir?

30) OhVilleLumière92 3 mars à 11:06

Aller les marseillaises vous pourrez toujours vous consoler et vous detendre sur un cd ou un morceau de votre fan numero 1 , le bien nommé mat pokora

Un phénomène nouveau, né grâce à Internet, consiste à " faire rage » en critiquant tout le monde et en semant des propos malveillants voire même parfois injurieux. Le « rageux » ne critique pas les autres parce qu'il possède des arguments, il le fait tout simplement par mauvaise foi malintentionnée. Ainsi, l'exemple 27 montre un échange où la conclusion ha bon et pourquoi pas ? est une belle illustration de méchanceté sans raison. Les « rageux » prononcent des propos médisants (exemple 28) ou ironiques (exemple 29), il arrive aussi qu'ils enchaînent les méchancetés pour passer leur nerfs sur quelqu'un (exemple 30).

31) laziale92 28 déc. à 15:07

Lui, il en a ou il faut !!!

Neoflash3 28 déc. à 15:36

Pas dans la tête en tout cas

32) BagGone1973 14 mars à 19:36

Aucune faute d'orthographe... quelque chose me dit que ça n'est pas lui qui a écrit...

33) edbergwaddle 20 janv. à 20:48

Il pourra toujours se consoler avec le hot d'or

34) olibest59 4 mars à 13:53

Ils ont oublié de mentionner aussi le big Mac volé :/

breizh 354 mars à 9:00

Oh M....! J'espère qu'on ne lui a pas volé ces ballons d'or...j'deconne, heureusement qu'il n'a gagné aucun trophée, il faut regarder le point positif être nul parfois ça a du bon.

JeanValjeandeToulon 3 mars à 8:19

Gignac a-t-il un alibi? on l'a pas vu hier sur le terrain

JLO75 3 mars à 8:13

Sa collection de jouets Macdo n'a pas été dérobée...

35) thomas5976 3 mars à 8:49

Juste avant, ils avaient été chez Valbuena. Mais ils n'ont trouvé que des trottinettes...

Certains footballeurs sont plus souvent la proie des internautes (voire des « rageux ») que d'autres, leurs capacités physiques ou intellectuelles sont le plus souvent visées. Les supporters de clubs adverses aiment critiquer les joueurs de leurs rivaux. Cela est peut être un acte de réconfort qui vise à neutraliser le danger sportif que représentent certains footballeurs et qui consiste à les tourner en ridicule. Les moqueries qui apparaissent dans les commentaires visent le plus 
souvent l'intelligence (exemple 31), le niveau d'éducation (exemple 32) ou le physique (exemples 34 et $35^{9}$ ). Les commentaires des internautes sont souvent adaptés au contenu de l'article, l'exemple 34 mentionne le cambriolage de la maison d'un joueur de l'Olympique de Marseille pendant le match de son équipe face au Paris Saint-Germain à l'extérieur. Le post affirmant qu'heureusement qu'il n'a gagné aucun trophée, il faut regarder le point positif être nul parfois ça $a d u$ bon est une bénédiction déguisée qui implique le manque de talent du joueur. Les moqueries peuvent aussi concerner des situations qui sont seulement connues des initiés. L'exemple 33 est une allusion au ballon d'or ${ }^{10}$ que Franck Ribéry n'a finalement pas obtenu, et le hot $\mathrm{d}^{\text {'or }}{ }^{11}$ se réfère à son rôle dans l'affaire Zahia.

36) Ludman 14 mars à 21:52

Il n'aurait du jamais la commencer cette tête en bois! Bon vent Pinochio!

37) panenka37 7 mars à 15:41

on en a rien a secouer de ton equipe de gloss

38) paris-go du 4216 juin à 12:34

Ta dernière phrase résume tout... Un FOOTIX, rien de plus...

39) kobultras 3 mars à $7: 42$

Des nouvelles du futur transfert de thauvin??? Il serait annoncer en excellence vu son niveau de jeu....

Pinpenade 3 mars à 9:11

Mais seulement dans un club de valeurs...

iv4n11 3 mars à 10:53

Bon on a pas entendu Thauvin. Ses valeurs à lui sont où? Qu'est ce qu'il a prouvé sur le terrain hier?

ipod92370 3 mars à 11:48

Il les cherché dans le couloir pour rentrer au vestiaire.

Les insultes envers les héros des articles sont plutôt rares, le fait qu'il soit interdit d'utiliser des mots considérés comme vulgaires en est peut-être la raison.

\footnotetext{
${ }^{9}$ Dans l'exemple 34, on fait référence au joueur de l'Olympique de Marseille André-Pierre Gignac qui a un physique plutôt solide d'où la moquerie qu'en cambriolant sa maison les voleurs ont oublié le Big Mac (qui connote un attribut des gens obèses). Dans le cas de l'exemple 35, il s'agit de Matthieu Valbuena un joueur de 1,63 m d'où la trottinette comme attribut d'un " petit » enfant.

${ }^{10}$ La prestigieuse récompense pour le meilleur joueur du monde attribuée par le magazine France Football.

${ }^{11}$ Récompense attribuée aux actrices et aux acteurs des films X, qui a été inspirée par la palme d'or de Cannes.
} 
Cependant dans le corpus recueilli, on trouve des appellations telles que Pinochio, gloss ou footix ${ }^{12}$ qui s'appliquent plutôt aux autres utilisateurs du site.

Il arrive parfois que les internautes s'acharnent sur un footballeur en raison de sa déclaration aux médias. Florian Thauvin (joueur de l'Olympique de Marseille) a déclaré dans une interview : "Quand on parle de Paris, on dit, c'est vrai, qu'ils ont de bons joueurs et une grande équipe. Mais il n'y a plus de valeurs au PSG. Ils ne parlent plus que d'argent » (interview dans le magazine Surface du 19 février 2014). Cette déclaration a bouleversé les supporteurs du club adverse qui ont commencé une campagne de dédain dirigée contre le footballeur. Dans l'exemple 39, mis à part la critique du geste, on peut observer la répétition du mot «valeur», ce qui a comme but de souligner le mot qui a déclenché le conflit.

40) b_careful 28 déc. à 16:27

Triste est la Valls que dansent les dirigeants français. Anelka leur passe le bonjour d'Outre-Manche.

41) sadiki 30 janv. à 8:53

hey les pros de la justice, on dit pas acquittement et non lieu mais une relaxe.

TerminatOL 30 janv. à 9:44

Aui ; d'ailleurs les 2 joueurs se sont sûrement bien relaxés !!... Enfin, faudrait leur demander...!

42) ks1977 20 janv. à 20:39

Sacré Frank je suis convaincu que tu aurais quand même préférer soulever le ballon d'or.

Waingro 20 janv. à 21:57

Bah mieux vaut soulever 2 ballons qu'un seul non?

43) mamad 114 août à 17:22

En prison tout de suite et ne passez pas par la case d ? part!!!

44) ekl3ktic 16 juin à 11:23

Vas y Francky, c'est bon! Vas y Francky, c'est bon bon bon!!

Les échanges de commentaires des internautes donnent parfois naissance à des jeux de mots ludiques. Le contexte de certains énoncés est favorable à des répliques virulentes, qui peuvent être des homophones comme le cas de l'exemple 40 où le nom du politicien Valls ${ }^{13}$ est utilisé dans le contexte d'une phrase où devrait figurer le mot désignant la danse - la valse. Dans l'exemple 41,

${ }^{12}$ C'était la mascotte de la coupe du monde France 98. En français familier, on dénomme comme ça une personne qui s'autoproclame expert dans le domaine du football, mais en réalité c'est une personne dilettante.

${ }^{13}$ Manuel Valls, premier ministre de la France à partir du 31 mars 2014. 
on retrouve le mot relaxe : la décision du tribunal est confondu avec la 'relax' de relaxation 'détente'. On retrouve aussi parfois des homonymes, comme c'est le cas de l'exemple 42, où le ballon (de foot) est confondu exprès avec son homographe ballon (sein par métaphore). Certains commentaires possèdent une valeur cryptique, afin de les décrypter, il est nécessaire de connaître la culture générale du pays. Ainsi dans l'exemple 43 apparaît l'expression ne pas passer par la case départ qui est une référence au fameux jeu Monopoly et l'exemple 44 fait référence à Franky (Franck Ribéry) et une chanson de Franky Vincent à forte connotation sexuelle - "Alice ça Glisse".

En guise de conclusion, il faut constater que les commentaires des internautes publiés sous les articles du site lequipe.fr sont des arguments subjectifs qui relèvent de la volonté d'exprimer leur point de vue sur le contenu de l'article ou de polémiquer avec d'autres internautes. Ces remarques et ces commentaires sont motivés par l'article ou les réactions des autres utilisateurs du site. Les émotions et les opinions personnelles sont manifestées dans les post, où l'on retrouve des commentaires singuliers ou des échanges à caractère critique, informatif, ludique et même haineux. L'anonymat qui est offert par Internet aide les gens à « se laisser aller », c'est-à-dire à s'exprimer librement sans avoir peur des conséquences et des répercussions. L'analyse du corpus de commentaires des internautes sur les articles liés au football, «...nécessite que soit prise en compte la dimension, sinon sa visée, argumentative » (Amossy, $2006: 248$ ), le site lequipe.fr est destiné aux fans de football, c'est pourquoi les non-initiés peuvent parfois avoir des problèmes avec la compréhension de certaines énonciations qui sont fortement liées au monde du football. 


\section{BIBLIOGRAPHIE}

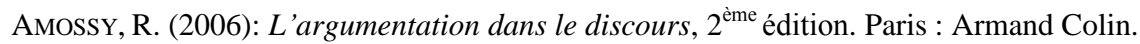

Charaudeau, P., Maingueneau, D. (2002): Dictionnaire d'analyse du discours. Paris : Seuil.

DrosiK, A. (2010): „W sieci Sieci. Jak Internet zmienia sferę publiczną”. In : (ed.) W. PiątkowskaStepaniak, A. Drosik, Władza mediów. Opole: Wydawnictwo Uniwersytetu Opolskiego, 117-130.

FONTANIER, P. (1977): Les figures du discours. Paris : Flammarion.

Galatanu, O., Cozma A.-M., BellachHab, A. (2012): « La force des mots : valeurs et violence dans les interactions verbales. Introduction ». Signes, Discours et Sociétés, 8. La force des mots : valeurs et violence dans les interactions verbale (www.revue-signes.info/document. php?id=2758)

LABOV, W. (1993): Le parler ordinaire. Paris : Les Éditions de Minuit.

Maingueneau, D. (1993): Le Contexte de l'auvre littéraire. Énonciation, écrivain, société. Paris : Dunod.

Martinet, A. (1996): Eléments de linguistique générale, $4^{\mathrm{ème}}$ édition. Paris : Armand Colin.

NunBerg, G. (1999): «Les enjeux linguistiques d'Internet ». Critique internationale, 4, 105-121.

Perelman, Ch. (2009): L'empire rhétorique. Paris : Librairie philosophique J. Vrin.

Plantin, CH. (1996): L'argumentation. Paris : Seuil. 